\title{
Agent-Based Modeling and Institutional Design ${ }^{1}$
}

Leigh Tesfatsion, Professor of Economics, Mathematics, and Electrical and Computer Engineering, Iowa State University, Ames, IA 50011-1070 USA.

Email: tesfatsi@iastate.edu, Web: www.econ.iastate.edu/tesfatsi/

\begin{abstract}
The recent economic crisis has led to calls for a comprehensive restructuring of energy, financial, health care, and educational systems. Critics worry the restructuring of these complex institutional arrangements could produce adverse unintended consequences. Given these concerns, pre-testing of proposed changes is eminently desirable but also exceedingly difficult. This essay focuses on the potential use of agent-based modeling for studying proposed changes in institutional arrangements in advance of actual implementation. Ongoing agent-based research on the restructuring of electric power markets is used for concrete illustration.
\end{abstract}

Key Words: Agent-based modeling, institutional design, electric power markets

JEL Codes: C6, L1, Q4

Latest Revision: 14 April 2010

\section{Introduction}

The standard approach to economic modeling entails the analytical specification of parameterized systems of equations. Using this approach, it is extraordinarily difficult to capture physical, institutional, and behavioral aspects of economies with empirical fidelity and still retain analytical tractability.

All of us have no doubt sat through many painful seminars in which economists have presented analytical models purporting to represent reality that would strike a typical person (and perhaps a good portion of the profession) as empirical nonsense. Yet our wont in the past has been to accept this as necessary because, after all, what else can be done. Equations must be solvable.

When the issue at hand is the explanation of an empirical regularity, the lack of micro-validated underpinnings can perhaps be glossed over by focusing audience attention on the matching of model outcomes to selected empirical data. For example, within mainstream macroeconomics today it is common to see researchers resorting to ad-hoc shocks, ad-hoc parameter calibrations, and/or incredible prior restrictions to ensure posterior model outcomes bend to the data.

On the other hand, when the focus of a study is an institution, a retreat to these time-honored tactics simply will not wash. The institution must be explicitly modeled. It is the centerpiece of the study from which eyes cannot be averted.

\footnotetext{
${ }^{1}$ Invited short essay for a special issue of the Eastern Economic Journal on agent-based modeling.
} 
What is to be done, then, when the issue of interest is institutional design and performance? Look out upon any actual socio-economic landscape. What is observed are not parameterized systems of equations but rather collections of autonomous people interacting through historical time. The interactions of these people are channeled and constrained by physical and institutional arrangements. In turn, these arrangements are affected at various time scales by the interactions of the people.

This is the social reality we all experience. Cannot we model social reality the way we actually experience it? For institutional studies, in particular, are we not bound as social scientists to model social reality the way we actually experience it?

In this essay I argue, in the affirmative, that economists and other social scientists can model social reality the way we experience it, as collections of autonomous people interacting through historical time. Specifically, I argue that Agent-Based Modeling $(A B M)$ permits this to be done with empirical fidelity and logical rigor.

Section 2 provides a brief summary of the ABM methodology as it specifically relates to institutional research. More general discussions focusing on the applicability of ABM for social science research can be found in Barr et al. [2008], Epstein [2006], and Tesfatsion [2006]. Annotated pointers to ABM research on institutional design and other application areas can be found at Axelrod and Tesfatsion [2010] and Tesfatsion [2010a]. For concrete illustration, Section 3 presents sample findings from ongoing $\mathrm{ABM}$ research focusing on the restructuring of electric power markets. Concluding remarks are given in Section 4.

\section{Agent-Based Modeling and Institutional Research}

Agent-Based Modeling (ABM) begins with assumptions about "agents" and their interactions and then uses computer simulation to generate histories that reveal the dynamic consequences of these assumptions. The agents in ABMs can range from passive physical world features with no cognitive function to individual and group decision makers with sophisticated learning and communication capabilities.

More precisely, each agent in an ABM is a software program encompassing data as well as methods that act on this data. Agents can have widely varying data and methods, thus permitting cross-sectional heterogeneity. In addition, agents can contain other agents as member data, thus permitting hierarchical constructions. The data and methods of any particular agent are encapsulated, in the sense that they can be partially or completely hidden from other agents. This encapsulation renders agents imperfectly predictable from the viewpoint of other agents in their computational world.

In the real world all calculations must be done by entities actually residing in the world. Agent encapsulation forces ABM researchers to respect this constraint. Procedures encapsulated in the methods of a particular agent can only be implemented using the particular resources available to that agent. Moreover, all procedures must be implemented through the methods of agents within the world. Thus, relative to traditional equation-based modeling, this encapsulation permits a more 
transparent and realistic representation of real-world systems composed of interacting distributed entities with limited information and computational capabilities.

Cognitive agents in ABMs can exhibit behavioral adaptation, goal-directed learning, social communication (talking with each other!), and the endogenous formation of interaction networks. A key aspect of these cognitive agents is their relative autonomy relative to traditionally modeled economic agents. Cognitive ABM agents can self-activate and self-determine their actions on the basis of internal data and methods hidden from other agents. Moreover, these internal data and methods can change over time as the cognitive agents explore and interact within their world.

Indeed, cognitive agents in ABMs should be able to pass the following constructive replacement test: Given any cognitive agent interacting within an $\mathrm{ABM}$ through its public interface (i.e., through its publicly accessible data and methods), it should be feasible to replace this agent with a person that interacts within the ABM through this same public interface. This leads naturally to the idea of a constructive Turing test for judging the empirical fidelity of cognitive agent representations: Would a person interacting within a mixed human-computational agent model be able to discern which other agents are human and which are computational? [Barr et al., 2008].

ABM researchers use controlled experimentation to investigate how large-scale effects arise from the micro-level interactions of dispersed cognitive agents operating within variously specified physical and institutional environments. The institutions of interest have ranged from macroeconomic policy rules to the microeconomic procurement processes of individual firms. In ABM research focusing specifically on institutional design and performance, two basic normative questions are typically addressed. First, does the institution promote efficient, fair, and orderly social outcomes over time, despite possible attempts by cognitive agents to game the institution for personal advantage? Second, under what conditions might the institution give rise to unintended consequences?

ABM experiments resemble wetware culture-dish experiments. Each ABM experiment starts from modeler-specified initial conditions and then proceeds forward in time without further modeler interventions.

For example, the first step in an ABM institutional study is to develop an experimental design for studying a particular institutional issue of interest. The second step is to construct a computational world ("culture dish") that incorporates salient features of the institution for the purpose at hand. This computational world should include cognitive agents that represent actual or projected real-world participants in these institutions. Ideally, these cognitive agents should face the same types of physical and financial constraints as their real-world counterparts.

The third step in an ABM institutional study is to specify a set of initial conditions for the computational world in accordance with the experimental design. Starting from these initial conditions, the fourth step is to permit the computational world to evolve over time driven solely by agent interactions, with no intervention from the modeler. The fifth step is to record outcomes of interest. The modeler then repeatedly iterates through these third, fourth, and fifth steps until all initial conditions specified under the experimental design have been tested. 


\section{Illustrative Application: Electricity Market Design}

In 2003 the U.S. Federal Energy Regulatory Commission (FERC) recommended the adoption of a common market design for U.S. wholesale power markets [FERC, 2003]. As indicated in Figure 1, and elaborated in Joskow [2006], versions of this design have been implemented (or adopted for implementation) in North American energy regions in the Midwest, New England, New York, the Mid-Atlantic States, California, the Southwest, and Texas.

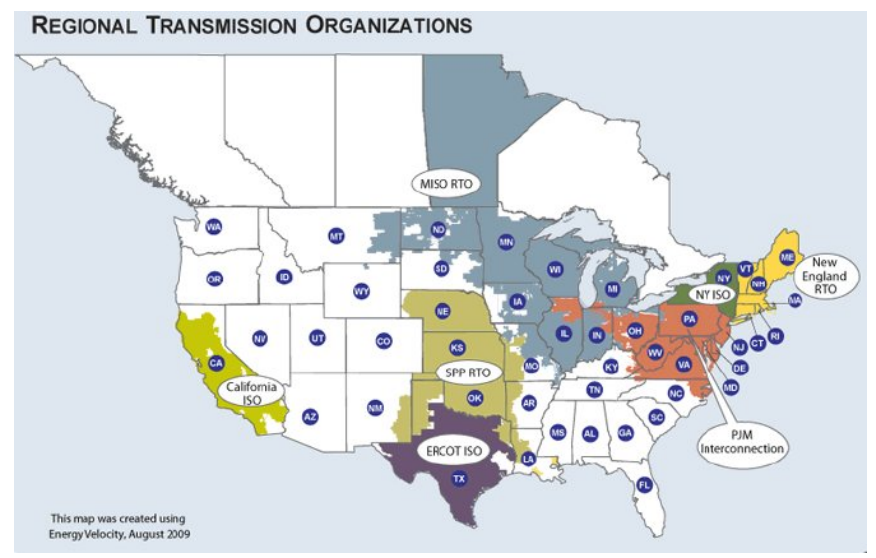

Fig. 1 North American energy regions that have adopted FERC's wholesale power market design. Source: www.ferc.gov/industries/electric/indus-act/rto/rto-map.asp

A core feature of FERC's design is a reliance on locational marginal pricing $(L M P)$ to manage transmission grid congestion. Under this pricing system, the price charged to wholesale buyers and received by wholesale sellers at a particular transmission grid bus location at a particular point in time is the least cost to the system of providing an additional increment of power at that bus location at that time.

For the past several years a group of researchers at Iowa State University has been developing and using an agent-based test bed AMES (Agent-based Modeling of Electricity Systems) to explore the performance characteristics of wholesale power markets operating under FERC's design [Tesfatsion, 2010b]. As indicated in Figure 2, AMES models strategic trading among a dispersed collection of Load-Serving Entities (LSES) who bid to buy power at wholesale and Generation Companies (GenCos) who offer to sell power at wholesale. These trading activities take place within a two-settlement (real-time and day-ahead) energy market system administered by a not-for-profit Independent System Operator (ISO), with congestion managed by LMP. The power flows resulting from these trading activites are constrained by the physical characteristics of an underlying $\mathrm{AC}$ transmission grid.

AMES experiments have been conducted using multi-period versions of a commonly used 5-bus training case and a standard 30-bus IEEE test case [Tesfatsion, 


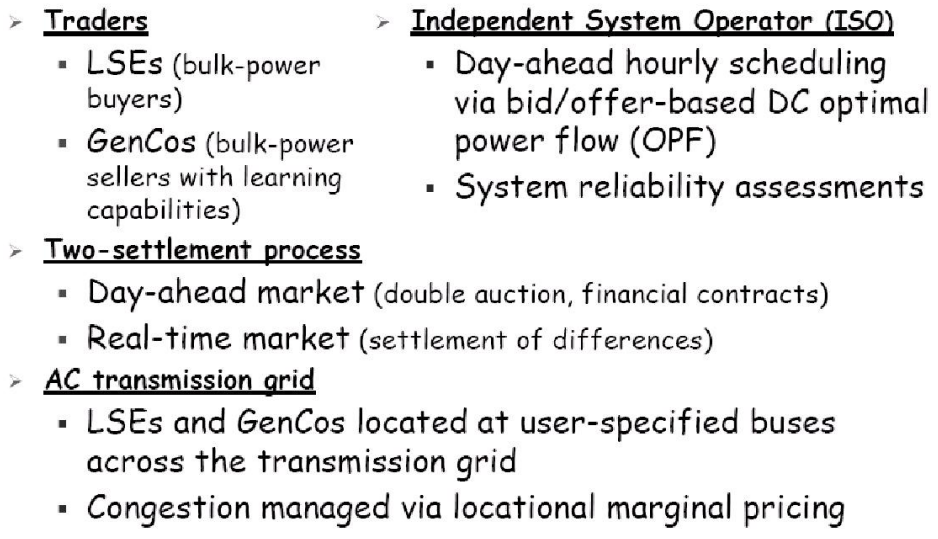

Fig. 2 Core features of FERC's wholesale power market design that have been incorporated into the AMES Wholesale Power Market Test Bed.

2010b]. Key experimental treatment factors have included GenCo learning capabilities, the form of GenCo supply offers, the price-sensitivity of LSE demand bids, and price caps imposed by the ISO for the mitigation of market power.

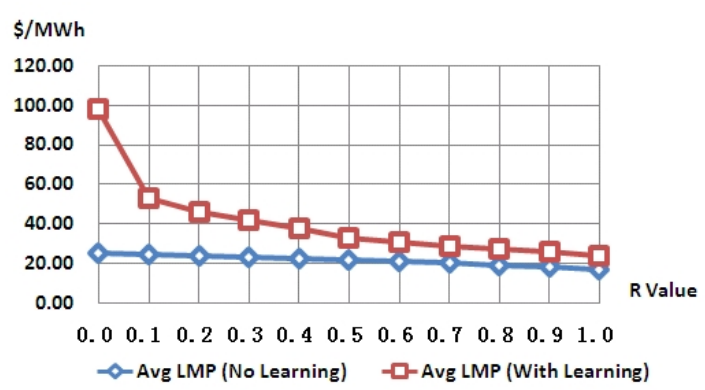

Fig. 3 Average LMP outcomes on day 1000 for a 5-bus test case with and without GenCo learning as the price-sensitivity of demand varies from $0 \%(\mathrm{R}=0.0)$ to $100 \%(\mathrm{R}=1.0)$.

One experimental finding has been the relative ease with which the GenCos can learn to exercise market power through economic and physical capacity withholding, even when LSE demand bids are 100\% price sensitive. As seen in Figure 3, this capacity withholding results in a rise in LMP levels that is particulary dramatic for treatments in which the price-sensitivity of demand is low.

Another experimental finding involves "ISO net surplus" collections in dayahead energy markets. ISO net surplus is determined each hour as the difference between the LMP payments received by the ISO from energy buyers (the LSEs) and the LMP payments distributed by the ISO to energy sellers (the GenCos). Congestion arising on a transmission grid in any hour necessarily results in separation 
between the LMPs at two or more transmission grid bus locations, which in turn necessarily results in a non-negative ISO net surplus for that hour.

As indicated by the experimental findings reported in Figures 3 through 5, ISO net surplus and GenCo net earnings are simultaneously enhanced in market circumstances unfavorable to market efficiency [Li and Tesfatsion, 2010]. Specifically, learning and price-sensitivity treatments resulting in greater GenCo capacity withholding, hence higher and more volatile LMPs, also result in greater ISO net surplus collections and GenCo net earnings. Moreover, these ISO net surplus collections and GenCo net earnings can be substantial in magnitude.

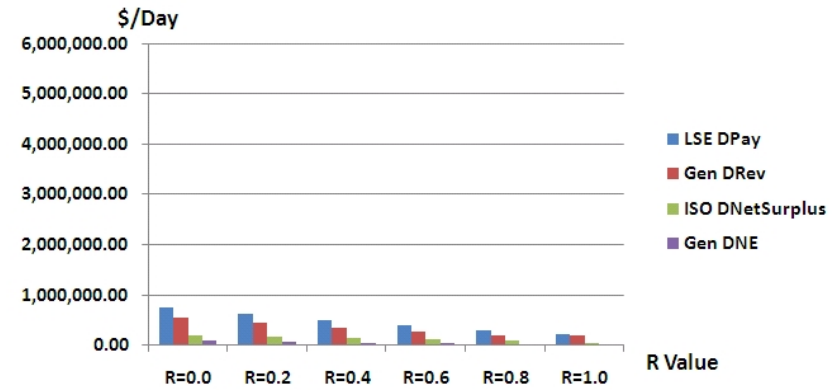

Fig. 4 LSE payments, GenCo revenues, ISO net surplus, and GenCo net earnings on day 1000 for the 5-bus test case without GenCo learning as the price-sensitivity of demand varies from $0 \%$ $(\mathrm{R}=0.0)$ to $100 \%(\mathrm{R}=1.0)$.

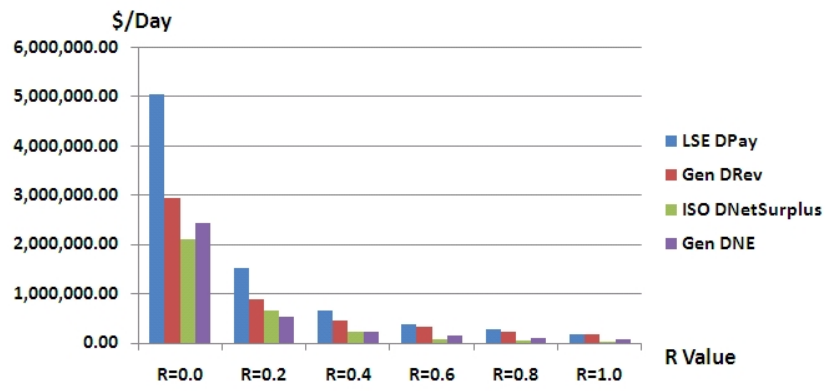

Fig. 5 Average LSE payments, GenCo revenues, ISO net surplus, and GenCo net earnings on day 1000 for the 5-bus test case with GenCo learning as the price-sensitivity of demand varies from $0 \%$ $(\mathrm{R}=0.0)$ to $100 \%(\mathrm{R}=1.0)$.

Empirical investigation confirms that ISO net surplus collections in actual North American energy regions operating under LMP can indeed be substantial [ $\mathrm{Li}$ and Tesfatsion, 2010]. For example, in 2008, ISO net surplus collections ranged from US\$121 million in New England (ISO-NE) to US\$2.66 billion in the Mid-Atlantic States (PJM). 


\section{Concluding Remarks}

Although this short essay has stressed ABM institutional research, it has highlighted several distinctive capabilities of ABM. First, ABM focuses on the playing out of processes over historical time rather than on the existence of equilibria. Second, the flexible nature of ABM permits researchers to incorporate complicated physical, institutional, and behavioral aspects of real-world situations. Third, ABM permits researchers to study the effects of changes in these aspects on outcome distributions, both spatially and temporally.

Given these distinctive capabilities, ABM is a welcome addition to the social science toolkit. However, the full potential of ABM will only be realized when supported by appropriate data collection permitting empirically-grounded modeling at the level of agent interactions.

Acknowledgements This work has been supported in part by grants from the Department of Energy at Pacific Northwest National Laboratory and the ISU Electric Power Research Center.

\section{References}

1. Axelrod, Robert, and Leigh Tesfatsion. 2010. Online Guide for Newcomers to Agent-Based Modeling in the Social Sciences, http://www.econ.iastate.edu/tesfatsi/abmread.htm

2. Barr, Jason M., et al. 2008. The Future of Agent-Based Research in Economics: A Panel Discussion. Eastern Economic Journal, 34:550-565.

3. Epstein, Joshua M. 2006. Generative Social Science: Studies in Agent-Based Computational Modeling, Princeton, NJ:Princeton University Press.

4. FERC, 2003. Notice of White Paper, U.S. Federal Energy Regulatory Commission, April.

5. Joskow, Paul. 2006. Markets for Power in the United States: An Interim Assessment, The Energy Journal, 27(1):1-36.

6. Li, Hongyan, and Leigh Tesfatsion. 2010. ISO Net Surplus Collection and Allocation in Wholesale Power Markets under LMP, Economics Working Paper No.09015, Iowa State University, Ames, IA, revised March.

7. Tesfatsion, Leigh. 2006. Agent-Based Computational Economics: A Constructive Approach to Economic Theory, in Handbook of Computational Economics: Volume 2, Agent-Based Computational Economics, edited by Leigh Tesfatsion and Kenneth L. Judd, Handbook in Economics Series, Amsterdam:North-Holland/Elsevier, 831-880

8. 2010a. Agent-Based Computational Economics Homepage, http://www.econ.iastate.edu/tesfatsi/ace.htm

9. 2010b. AMES Wholesale Power Market Test Bed Homepage, http://www.econ.iastate.edu/tesfatsi/AMESMarketHome.htm 